\title{
Risk factors for poor treatment outcomes of 2266 multidrug-resistant tuberculosis cases in Ho Chi Minh City: a retrospective study
}

Le Hong Van ${ }^{1 *}$ DD, Phan Trieu Phu', Dao Nguyen Vinh, Vo Thanh Son ${ }^{1}$, Nguyen Thi Hanh ${ }^{1}$, Le Thanh Hoang Nhat ${ }^{1}$, Nguyen Huu Lan², Truong Van Vinh², Nguyen Thi Mai Trang ${ }^{2}$, Dang Thi Minh Ha², Guy E. Thwaites ${ }^{1,3}$ and Nguyen Thuy Thuong Thuong ${ }^{1 *}$

\begin{abstract}
Background: Multidrug resistant tuberculosis (MDR-TB) remains a serious public health problem with poor treatment outcomes. Predictors of poor outcomes vary in different regions. Vietnam is among the top 30 high burden of MDR-TB countries. We describe demographic characteristics and identify risk factors for poor outcome among patients with MDR-TB in Ho Chi Minh City (HCMC), the most populous city in Vietnam.

Methods: This retrospective study included 2266 patients who initiated MDR-TB treatment between 2011 and 2015 in HCMC. Treatment outcomes were available for 2240 patients. Data was collected from standardized paper-based treatment cards and electronic records. A Kruskal Wallis test was used to assess changes in median age and body mass index (BMI) over time, and a Wilcoxon test was used to compare the median BMl of patients with and without diabetes mellitus. Chi squared test was used to compare categorical variables. Multivariate logistic regression with multiple imputation for missing data was used to identify risk factors for poor outcomes. Statistical analysis was performed using R program.

Results: Among 2266 eligible cases, $60.2 \%$ had failed on a category I or II treatment regimen, 57.7\% were underweight, 30.2\% had diabetes mellitus and 9.6\% were HIV positive. The notification rate increased 24.7\% from 2011 to 2015. The treatment success rate was $73.3 \%$. Risk factors for poor treatment outcome included HIV co-infection (adjusted odds ratio (aOR): 2.94), advanced age (aOR: 1.45 for every increase of 5 years for patients 60 years or older), having history of MDR-TB treatment (aOR: 5.53), sputum smear grade scanty or 1+ (aOR: 1.47), smear grade 2+ or 3+ (aOR: 2.06), low BMl (aOR: 0.83 for every increase of $1 \mathrm{~kg} / \mathrm{m} 2$ of BMl for patients with $\mathrm{BMl}<21$ ).

Conclusion: The number of patients diagnosed with MDR-TB in HCMC increased by almost a quarter between 2011 and 2015. Patients with HIV, high smear grade, malnutrition or a history of previous MDR-TB treatment are at greatest risk of poor treatment outcome.
\end{abstract}

Keywords: Multidrug resistant tuberculosis, Retrospective, Treatment outcome, Risk factors, Vietnam

\section{Background}

Multidrug resistant tuberculosis (MDR-TB), defined as tuberculosis (TB) with resistance to at least rifampicin and isoniazid, is a serious public health problem. In 2017, there were an estimated 558,000 incident cases and 230,000 deaths due to MDR/Rifampicin resistant (RR)-TB worldwide. Treatment of MDR-TB is lengthy,

\footnotetext{
*Correspondence: vanlh@oucru.org; thuongntt@oucru.org

'Tuberculosis group, Oxford University Clinical Research Unit, 764 Vo Van Kiet street, District 5, Ho Chi Minh City, Vietnam

Full list of author information is available at the end of the article
}

toxic and expensive, with success rates reported globally at $55 \%$ in 2017 [1]. HIV co-infection, low body mass index (BMI) and positive sputum smear are predictors of poor MDR-TB outcomes, but the effects of these predictors may vary in different regions [2-6].

Vietnam is listed by World Health Organization as having a high TB and MDR-TB burden. The estimated incidence of TB in 2017 was 129 per 100,000 people. A national survey in 2011 showed resistance to any drug was $32.7 \%$ in new TB patients and $54.2 \%$ in previously treated patients [7]. The prevalence of

(c) The Author(s). 2020 Open Access This article is distributed under the terms of the Creative Commons Attribution 4.0 International License (http://creativecommons.org/licenses/by/4.0/), which permits unrestricted use, distribution, and 
MDR-TB was $4.1 \%$ in new patients and $17 \%$ in previously treated patients, with 4900 estimated new cases annually countrywide [1]. Ho Chi Minh City (HCMC) is the most populous city in Vietnam (around 8 million people) and also the center for TB and drugresistant TB management in Southern Vietnam. In 2009, the Vietnam National TB Programme initiated the Programmatic Management of Drug-resistant TB (PMDT) under the support of Global Fund (Switzerland) to provide free treatment and support for MDR-TB patients [2]. Pham Ngoc Thach hospital (PNTH) is a tertiary referral center for TB and lung diseases in Southern Vietnam, which provided treatment for $81 \%$ of registered MDR-TB patients in Vietnam in 2010 [8]. The number of patients enrolled in PMDT rapidly increased from 2010 to 2014; yet, the total number of MDR-TB patients enrolled for treatment in Vietnam in 2014 was still very low, with only a third of the estimated 5100 cases receiving treatment [2]. From 2011, all 24 district TB units (DTUs) of HCMC participated in the MDR-TB management. However, until now, there has been no information about the notification trend, treatment outcome and factors associated with poor treatment outcome of MDR-TB patients in HCMC. In this study, we retrospectively investigated the demographic characteristics and risk factors for poor treatment outcomes of MDR-TB in HCMC from 2011 to 2015.

\section{Methods}

\section{Study setting and population}

Patients were hospitalized in PNTH for 7 to 14 days to initiate treatment, and then referred to DTUs for outpatient follow-up. Treatment modalities did not change during the study time, with the standardized combination of 6 drugs for a total of 18 to 24 months of treatment [2]. Table A in supplementary data outlines the standardized treatment regimens used in the study time and Table B describes the treatment outcomes of MDR-TB.

Sputum samples from suspected MDR-TB patients or from patients with MDR/RR-TB detected by XpertMTB/ RIF or line probe assay (LPA) were sent to PNTH to confirm MDR-TB by phenotypic drug susceptibility testing (DST). Culture and DST in solid and liquid media was the main diagnostic method prior 2012. Although pyrazinamide and ethambutol were part of the standardized MDR-TB regimen, detecting resistance to these drugs was expected to be challenging due to the poor performance of phenotypic DST assays [9]. GenoType MTBDRplus (Hain Lifescience GmbH, Germany - LPA) and Xpert MTB/RIF (Cepheid, USA) were introduced to detect MDR/RR-TB in 2010 and
2012 respectively. We later use the term "DST" to refer to phenotypic DST in our results.

We included all patients who initiated treatment with second-line drugs in HCMC under PMDT from January 2011 to December 2015. To ensure that all treatment outcomes could be captured, more recently diagnosed cases were excluded. Patients were excluded if 1) MDR-TB was diagnosed by a molecular assay but DST reported rifampicin or isoniazid susceptibility; 2) they were enrolled in the STREAM trial [10] to receive a 9-month regimen; 3) they did not start treatment (Fig. 1).

\section{Data collection}

Demographic and clinical information, radiographs, acid-fast bacilli (AFB) staining, DST results, treatment regimens and treatment outcomes were recorded into structured paper forms. To improve reliability, we collected data from both standardized paper-based treatment cards and electronic records and verified data during the data collection, entry and analysis processes.

\section{Statistical analysis}

Data analysis was performed using $\mathrm{R}$ program version 3.5.2 [11]. The baseline characteristics were summarized in terms of the number of cases (percentage) for categorical variables, and median with interquartile range (IQR) for continuous variables. We used a Kruskal Wallis test assess changes in median age and BMI over 5 years, and a Wilcoxon test to compare the median BMI of patients with and without diabetes mellitus (DM). Chi squared tests were used to compare categorical variables.

Multivariate logistic regression was used to identify risk factors contributing to poor treatment outcome. Treatment outcome was binary, defined as "success" (cured, completed) or "non-success" (death, failure, lost to follow-up) as in [12]. HIV co-infection, history of previous MDR-TB treatment, AFB smear grade and BMI were included as covariates in the model. Gender, age and DM status were adjusted for as potential confounders. The covariates age and BMI were modelled in a piecewise linear form. For age we used an index variable (age $\leq 60$ years old) and a linear pattern for age greater than 60 years old (Figure A). Similarly for BMI, we used an index variable $\left(B M I \geq 21 \mathrm{~kg} / \mathrm{m}^{2}\right.$ ) and a linear pattern for BMI less than $21 \mathrm{~kg} / \mathrm{m}^{2}$ (Figure B). To minimize potential bias from missing data, we used multiple imputation by chained equation ("mice" package in R [13]) and performed multivariate logistic regression models using both imputed data analysis and complete case analysis. 


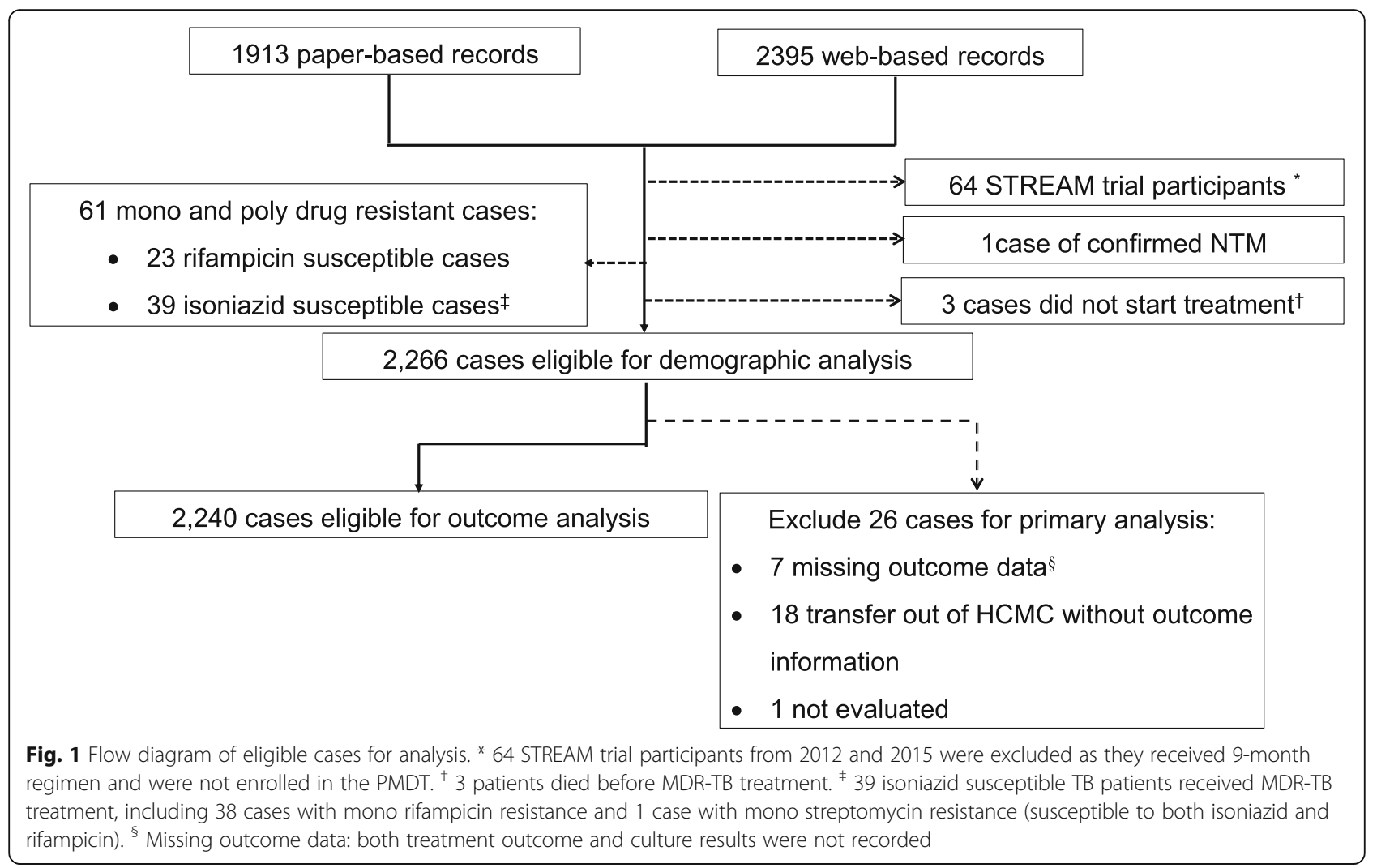

\section{Results}

\section{Characteristics of MDR/RR-TB patients}

Two thousand three hundred ninety-five electronic records and 1913 paper-based records were available and 2266 MDR/RR-TB cases were included (Fig. 1). Of these patient diagnosed between 2011 and 2015, eight patients relapsed, 13 were re-treated after loss to follow-up, and two were re-treated after treatment failure. Baseline characteristics are presented in Table 1. Median age among the 2266 cases was 43 years (IQR: $33-53$ years) and did not change between 2011 and $2015(p=0.481)$. A total of 204 patients ( $9.6 \%$ of tested patients) were HIV co-infected; of these, 33 (16.1\%) were registered as new TB patients and 21 (10.3\%) had extra-pulmonary MDR-TB, including 10 (4.9\%) patients with MDR-TB meningitis. MDR-TB meningitis was more common than in patients without HIV co-infection $(p<0.001)$. Eighty four of 204 HIV co-infected patients $(41.2 \%)$ were established on antiretroviral therapy (ART) before starting MDR-TB treatment. The remaining HIV co-infected patients $(58.8 \%)$ started ART at least 2 weeks after starting MDR-TB treatment. Among 1815 cases for whom BMI data were available, $57.8 \%$ was classified as underweight and $25.1 \%$ severely underweight. Median BMI did not change over 5 years $(p=0.966)$. DM status was available for 1189 patients (52.5\%), 359 of whom (30.2\%) had DM. Median BMI among patients with DM (20.0 $\mathrm{kg} / \mathrm{m}^{2}$ ) was higher than among patients without DM $\left(17.8 \mathrm{~kg} / \mathrm{m}^{2}\right)(p<0.001)$ and HIV co-infection in patients with DM $(0.9 \%)$ was lower than among patients without $\operatorname{DM}(9.8 \%)(p<0.001)$.

\section{Drug resistance pattern}

Table 2 outlines the observed drug resistance patterns. DST results were retrieved for 502 isolates from $490 \mathrm{pa}-$ tients. Ten patients had results for two isolates at different time points, and one patient had results for three isolates. Among the 490 patients with a DST result, 55.0 and $63.1 \%$ had resistance to pyrazinamide and ethambutol, respectively. Resistance to fluoroquinolones and injectable agents was seen in 12.7 and $8.1 \%$ of isolates, respectively. Among 378 patients with DST to secondline drugs, 63 (16.7\%) had pre extensively drug-resistant (XDR) TB and 8 (2.1\%) had XDR-TB.

\section{MDR-TB trend}

Figure 2 shows an increasing temporal trend from 2011 to 2015 for both the absolute number of cases diagnosed and the notification rate per 100,000 population. Numbers of notified MDR/RR-TB patients decreased by $9 \%$ between 2011 and 2012, and increased an average of $15.9 \%$ annually from 2012 to 2015 . The number of 
Table 1 Characteristics of MDR-TB patients in HCMC from 2011 to 2015

\begin{tabular}{|c|c|}
\hline Characteristic & n (\%) \\
\hline Total & 2266 \\
\hline Age at diagnosis (years, median (IQR)) & $43(33-53)$ \\
\hline$>60$ years old & $197(8.7 \%)$ \\
\hline 18-60 years old & $2037(89.9 \%)$ \\
\hline$<18$ years old & $32(1.4 \%)$ \\
\hline Male & $1715(75.7 \%)$ \\
\hline \multicolumn{2}{|l|}{ Site of disease } \\
\hline Pulmonary & 2237 (98.7\%) \\
\hline Multi-organ ${ }^{a}$ & $37(1.7 \%)$ \\
\hline Extra pulmonary & $66(3 \%)$ \\
\hline Lymphadenitis & 22 \\
\hline Meningitis & 21 \\
\hline Pleuritis & 8 \\
\hline Bone and vertebral & 4 \\
\hline Soft tissue & 1 \\
\hline Gastro-intestinal & 1 \\
\hline \multicolumn{2}{|l|}{ Registration group } \\
\hline New & $128(5.6 \%)$ \\
\hline Relapse & $678(29.9 \%)$ \\
\hline Failure of regimen 1 & $512(22.6 \%)$ \\
\hline Failure of regiment 2 & $852(37.6 \%)$ \\
\hline Treatment after lost to follow-up & $47(2.1 \%)$ \\
\hline Transfer & $1(0 \%)$ \\
\hline Other & $46(2 \%)$ \\
\hline Not recorded & $2(0.1 \%)$ \\
\hline \multicolumn{2}{|l|}{ Regimen of previous treatment } \\
\hline । & $913(40.3 \%)$ \\
\hline$\|$ & $1012(44.6 \%)$ \\
\hline III & $2(0.1 \%)$ \\
\hline IV & $54(2.4 \%)$ \\
\hline No history of previous treatment & $128(5.6 \%)$ \\
\hline Unclear history & $157(7 \%)$ \\
\hline BMI at diagnosis $\left(\mathrm{kg} / \mathrm{m}^{2}\right)$ & $n=1815$ \\
\hline Median (IQR) & $17.86(15.76-19.96)$ \\
\hline Overweight (BMI $\geq 25$ ) & $46(2.5 \%)$ \\
\hline Normal BMI (BMI: 18.5-<25) & $721(39.7 \%)$ \\
\hline Mild underweight (BMI: 17-<18.5) & $343(18.9 \%)$ \\
\hline Moderate underweight (BMI: 16-<17) & $250(13.8 \%)$ \\
\hline Severe underweight $(\mathrm{BMI}<16)$ & $456(25.1 \%)$ \\
\hline HIV positive & 204/2136 tested for HIV (9.6\%) \\
\hline Diabetes & $359 / 1189(30.2 \%)$ \\
\hline Unknown history of diabetes & $1077(47.5 \%)$ \\
\hline \multicolumn{2}{|l|}{ Initial Diagnosis Method } \\
\hline DST & 274 (12.1\%) \\
\hline
\end{tabular}


Table 1 Characteristics of MDR-TB patients in HCMC from 2011 to 2015 (Continued)

\begin{tabular}{|c|c|}
\hline Characteristic & n (\%) \\
\hline Xpert & $1276(56.3 \%)$ \\
\hline Hain & 705 (31.1\%) \\
\hline DST of last treatment episode & $7(0.3 \%)$ \\
\hline Missing data & $4(0.2 \%)$ \\
\hline \multicolumn{2}{|l|}{ AFB smear at baseline } \\
\hline Positive & $1748(77.1 \%)$ \\
\hline$<1+$ & $158(9 \%)$ \\
\hline $1+$ & $895(51.1 \%)$ \\
\hline $2+$ & $357(20.5 \%)$ \\
\hline $3+$ & $275(15.7 \%)$ \\
\hline Unknown grade & $63(3.7 \%)$ \\
\hline Negative & $475(21 \%)$ \\
\hline Not recorded & $43(1.9 \%)$ \\
\hline \multicolumn{2}{|l|}{ Culture at diagnosis } \\
\hline Positive & $1371(60.5 \%)$ \\
\hline Negative & $178(7.8 \%)$ \\
\hline Non-Tuberculosis Mycobacterium (but Xpert positive) ${ }^{b}$ & $4(0.2 \%)$ \\
\hline Contaminated & $19(0.8 \%)$ \\
\hline Not recorded & 694 (30.6\%) \\
\hline
\end{tabular}

a involved both pulmonary and extra pulmonary TB

${ }^{\mathrm{b}}$ All four cases of culture positive for Non-Tuberculosis Mycobacterium also had GeneXpert detected Mycobacterium tuberculosis

MDR-TB cases and the notification rate increased 41.0 and $24.7 \%$ from 2011 to 2015 , respectively.

\section{Treatment outcomes}

Table 3 summarizes the treatment outcomes of 2240 MDR-TB patients whose treatment outcomes were retrievable. Successful outcomes were achieved in 1641

Table 2 Frequency of first and second-line drug resistance of MDR-TB in HCMC, 2011-2015

\begin{tabular}{ll}
\hline Drug resistance & $\mathrm{n} /$ total tested (\%) \\
\hline Patients with DST result ${ }^{\mathrm{a}}$ & 490 \\
First line drugs & \\
Pyrazinamide & $210 / 382(55.0 \%)$ \\
Ethambutol & $298 / 472(63.1 \%)$ \\
$\quad$ Streptomycin & $438 / 455(96.3 \%)$ \\
Second-line drugs & \\
Fluoroquinolones & \\
Any injectable agents & \\
All injectable agents & $48 / 378(12.7 \%)$ \\
Cycloserine & $31 / 384(8.1 \%)$ \\
Ethionamide/Prothionamide & $9 / 115(7.8 \%)$ \\
\hline
\end{tabular}

${ }^{\mathrm{a}}$ A total of 502 DST of 490 patients were retrievable

b fluoroquinolones include moxifloxacin, levofloxacin, ofloxacin

c injectable agents include kanamycin, amikacin and capreomycin
(73.3\%) patients, including 55.6\% who were cured and $17.7 \%$ who completed treatment but for whom data on cure were unavailable. Among those with unsuccessful outcomes, $10.1 \%$ died, $5 \%$ failed treatment and $11.6 \%$ were lost to follow-up. Patient characteristics by treatment outcome are further described in the supplementary material (Table C). 49/204 patients with HIV died (23.0\%), 8 (3.9\%) failed treatment and 42 (20.5\%) were lost to follow-up. Ten of 21 (47.6\%) patients with TB meningitis had successful outcomes, nine (42.6\%) died and two $(9.5 \%)$ were lost to follow-up. Among the 64 patients with pre-XDR-TB, 53.1\% had a successful outcome, $14.1 \%$ died, $23.4 \%$ failed treatment and $7.8 \%$ were lost to follow-up. Of 8 XDR-TB patients, 1 (12.5\%) was cured with a bedaquiline-containing regimen, 2 (25\%) died, including 1 who received a bedaquiline-containing regimen, and 5 (62.5\%) failed.

Of 259 patients lost to follow-up, median treatment duration was 200 days (IQR: $60-340$ ) with $56 \%$ lost during intensive phase. $17.3 \%$ had HIV co-infection, $32 \%$ had a positive AFB smear and $35.9 \%$ had a positive culture prior to being lost to follow-up.

\section{Risk factors for poor outcomes}

We evaluated the association between poor treatment outcome and HIV co-infection, history of previous 


\section{MDR-TB TREND DURING 2011-2015}

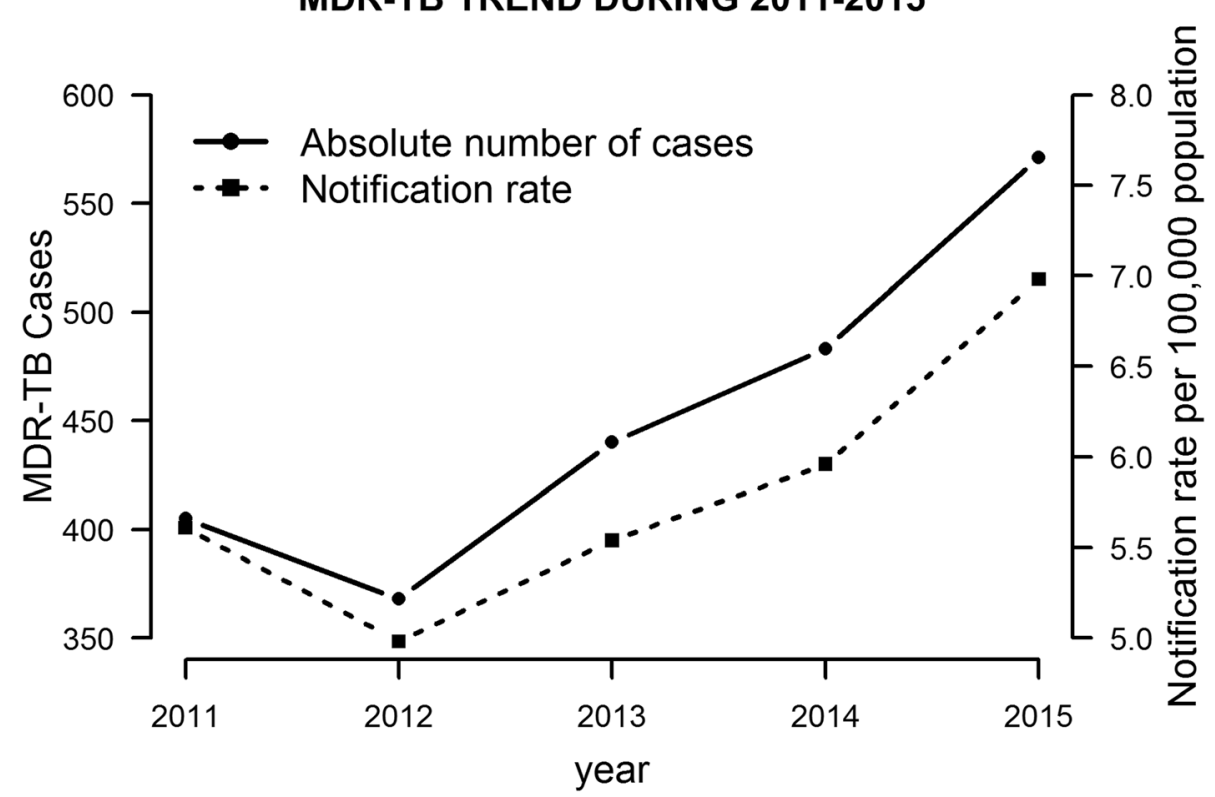

Fig. 2 MDR-TB trend for a 5 year period. The absolute number of MDR-TB cases are showned in the solid line, and the notification rate per 100,000 population in the dashed line

MDR-TB treatment, AFB smear grade and BMI. Male gender, age and DM status were included as in the multivariate logistic regression model as potential risk factors. Further analysis failed to show the interaction between HIV co- infection and other risk factors (age, gender, AFB smear grade, BMI, DM status and history of previous MDR-TB treatment) $(p=0.93)$. Since MDRTB patients received standardized treatment in 24 different DTUs, we did not include treatment site covariate in our final logistic regression model.

There were only small differences in the results between complete case and multiple imputation analysis (Table 4). Therefore, we presented the Forrest plot of results from the imputed data analysis (Fig. 3).

Independent risk factors for poor outcomes were older age (OR for every increase of 5 years when patients are older than 60: 1.45 , 95\% CI: 1.14-1.79, $p<0.001)$, HIV co-infection (OR: 2.94, 95\% CI: 2.07-4.16, $p<0.001$ ), a history of MDR-TB treatment (OR: 5.53, 95\% CI: $2.85-$ 10.72, $p<0.001$ ), AFB positive (OR: 1.47 for low smear grade $(1+$ and $<1+), 95 \%$ CI: $1.08-2.00, p=0.01$ and OR: 2.06 for high smear grade $(2+$ and $3+)$, 95\%CI: 1.49 $2.87, p<0.001$ ), and low BMI (OR: 0.83 for every increase of $1 \mathrm{~kg} / \mathrm{m} 2$ for patients with $\mathrm{BMI}<21,95 \% \mathrm{CI}$ : $0.79-0.87, p<0.001$ ) (Fig. 3).

\section{Discussion}

This is the first study to describe the characteristics and identify the risk factors for poor outcomes of MDR-TB in $\mathrm{HCMC}$, Vietnam. Although the incidence of $\mathrm{TB}$ in Vietnam has been declining [1], MDR-TB incidence is increasing. There was a shortage of Hain tests in the late 2011 and early 2012, which caused the drop in notified cases (information from annual report of HCMC TB program 2011-2012). The introduction of Xpert at the end of 2012 together with changes in MDR-TB

Table 3 Treatment outcomes of 2240 MDR-TB patients in HCMC, 2011-2015

\begin{tabular}{lllllll}
\hline Treatment outcome & $2011 \mathrm{n}(\%)$ & $2012 \mathrm{n}(\%)$ & $2013 \mathrm{n}(\%)$ & $2014 \mathrm{n}(\%)$ & $2015 \mathrm{n}(\%)$ & Total $\mathrm{n}(\%)$ \\
\hline Total $(\mathrm{n})$ & 405 & 365 & 438 & 476 & 556 & 2240 \\
Cured & $246(60.7 \%)$ & $190(52.1 \%)$ & $225(51.4 \%)$ & $289(60.7 \%)$ & $296(53.2 \%)$ & $1246(55.6 \%)$ \\
Completed & $64(15.8 \%)$ & $80(21.9 \%)$ & $85(19.4 \%)$ & $71(14.9 \%)$ & $96(17.3 \%)$ & $396(17.7 \%)$ \\
Died & $27(6.67 \%)$ & $39(10.7 \%)$ & $45(10.3 \%)$ & $51(10.7 \%)$ & $64(11.5 \%)$ & $226(10.1 \%)$ \\
Failed & $19(4.69 \%)$ & $20(5.48 \%)$ & $23(5.25 \%)$ & $22(4.62 \%)$ & $29(5.22 \%)$ & $113(5 \%)$ \\
Lost to follow-up & $49(12.1 \%)$ & $36(9.86 \%)$ & $60(13.7 \%)$ & $43(9.03 \%)$ & $71(12.8 \%)$ & $259(11.6 \%)$ \\
\hline
\end{tabular}


Table 4 Comparison of multivariate logistic regression models using complete case and multiple imputation analysis

\begin{tabular}{|c|c|c|c|c|c|c|}
\hline \multirow[t]{2}{*}{ Risk factors } & \multirow{2}{*}{$\begin{array}{l}\text { Successn } \\
(\%)\end{array}$} & \multirow{2}{*}{$\begin{array}{l}\text { Non- } \\
\text { success n } \\
\text { (\%) }\end{array}$} & \multicolumn{2}{|c|}{ Complete case analysis $^{a}$} & \multicolumn{2}{|c|}{ Multiple imputation analysis } \\
\hline & & & $\mathrm{aOR}$ & $95 \% \mathrm{Cl}$ & $\mathrm{aOR}$ & $95 \% \mathrm{Cl}$ \\
\hline Gender: male & $1231(75.0 \%)$ & $471(78.8 \%)$ & 1.55 & $0.99-2.42$ & 1.10 & $0.84-1.44$ \\
\hline Age $\leq 60$ years & & & 0.98 & $0.90-1.07$ & 1.01 & $0.96-1.06$ \\
\hline \multirow[t]{2}{*}{ Age $>60$ years } & & & 1.79 & $1.26-2.54$ & 1.45 & $1.14-1.79$ \\
\hline & \multicolumn{4}{|c|}{ For every increase of 5 years of age } & & \\
\hline Diabetes & $285(34.1 \%)$ & $71(21.3 \%)$ & 0.84 & $0.55-1.31$ & 0.81 & $0.61-1.08$ \\
\hline HIV positive & $102(6.5 \%)$ & 99 (17.9\%) & 3.12 & $1.66-5.84$ & 2.94 & $2.07-4.16$ \\
\hline History of previous MDR-TB treatment & $18(1.2 \%)$ & $38(7.0 \%)$ & 20.37 & $5.52-75.17$ & 5.53 & $2.85-10.72$ \\
\hline Low smear grade ${ }^{b}$ & $788(48.9 \%)$ & $259(44.1 \%)$ & 1.72 & $1.00-2.95$ & 1.47 & $1.08-2.00$ \\
\hline High smear grade ${ }^{c}$ & $416(25.8 \%)$ & $209(35.6 \%)$ & 2.25 & $1.28-3.93$ & 2.06 & $1.49-2.87$ \\
\hline AFB positive unknown & $40(2.5 \%)$ & $22(3.7 \%)$ & 2.70 & $1.08-6.78$ & 2.80 & $1.47-5.36$ \\
\hline $\mathrm{BMI}<21$ & & & 0.82 & $0.76-0.89$ & 0.83 & $0.79-0.87$ \\
\hline \multirow[t]{2}{*}{$\mathrm{BMI} \geq 21$} & & & 0.96 & $0.81-1.15$ & 1.06 & $0.93-1.2$ \\
\hline & \multicolumn{4}{|c|}{ For every 1 increase of BMI } & & \\
\hline
\end{tabular}

a Complete case analysis: non-imputed data

${ }^{b}$ Low smear grade: scanty or $1+$ on AFB smear

c High smear grade: $2+$ or $3+$ on AFB smear

diagnostic policies might have contributed to the increase in notified cases. Transmission of drug-resistant TB may also have contributed. With the rollout of Xpert, all smear positive patients could be screened for drug resistance since the end of 2015. The high rates of failure of regimen $1(22.6 \%)$ and regimen $2(37.6 \%)$ in patients eventually diagnosed with MDR-TB reflect previous inadequate screening for drug resistance among new and retreated patients, and highlight the importance of drug resistance screening for all $\mathrm{TB}$ patients regardless of their TB history.

In this RR/MDR-TB cohort, almost all strains were resistant to streptomycin (96.3\%), as previously observed in Vietnam [14-16]. This could be explained by the fact that the streptomycin was widely used in regimen 1 and 2 during the intensive phase for both new and retreated patients.

We found high rates of resistance to pyrazinamide (55.0\%) and ethambutol (63.1\%) in our MDR-TB cohort, as also reported by other studies $[17,18]$. This may reflect the fact that the majority of MDR-TB patients (94.3\%) already had exposure to first line anti-TB drugs and might have developed resistance to pyrazinamide and ethambutol during previous treatment. This will have limited the effectiveness of the standardized MDRTB regimen [19] and emphasizes the need for an approved genotypic DST to rapidly detect pyrazinamide resistance.

Resistance rates to fluoroquinolones (12.7\%) and injectable agents $(8.1 \%)$ were comparable to those seen in Vietnam in 2011 [20] but lower than in South Korea
[17] and average global rates [1]. Although these drugs are not used in the regimen 1 or 2 , the high rates of resistance might be explained by easy access to antibiotics without prescription in Vietnam [21].

HIV co-infection, positive baseline AFB smear, older age and previous treatment with second-line drugs are main risk factors for poor treatment outcomes in our cohort, which were also observed in cohorts in Estonia, Latvia, Philippines, Russia, Peru [4], and Ukraine [3]. Malnutrition was common (57.8\%) and a risk factor for poor outcome (OR: 0.81 for every $1 \mathrm{~kg} / \mathrm{m}^{2}$ increase of $\mathrm{BMI})$. Low BMI might be a consequence of severe disease and low socio-economic status, which are wellknown risk factors for poor outcome in TB. PMDT should focus on nutritional support to improve treatment outcomes.

The prevalence of DM in our cohort (30.2\%) was double that among patients with TB (13.7\%) in Hanoi, Vietnam [22] and was almost 6 times higher than among the general Vietnamese population in 2013 (5.4\%) [23]. Although DM is a known risk factor for poor treatment outcome in TB, for developing MDR$\mathrm{TB}$ and for reducing sputum conversion rate during MDR-TB treatment [24-26], it remains controversial whether DM also leads to poor treatment outcome of MDR-TB [24, 27, 28]. After adjustment for other factors, DM was not an independent risk for poor outcomes in our cohort, which agrees with pooled data analysis from cohorts in Latvia, Korea and Italy [29]. Due to the unavailability of DM treatment information, we do not know whether the effect of DM on 


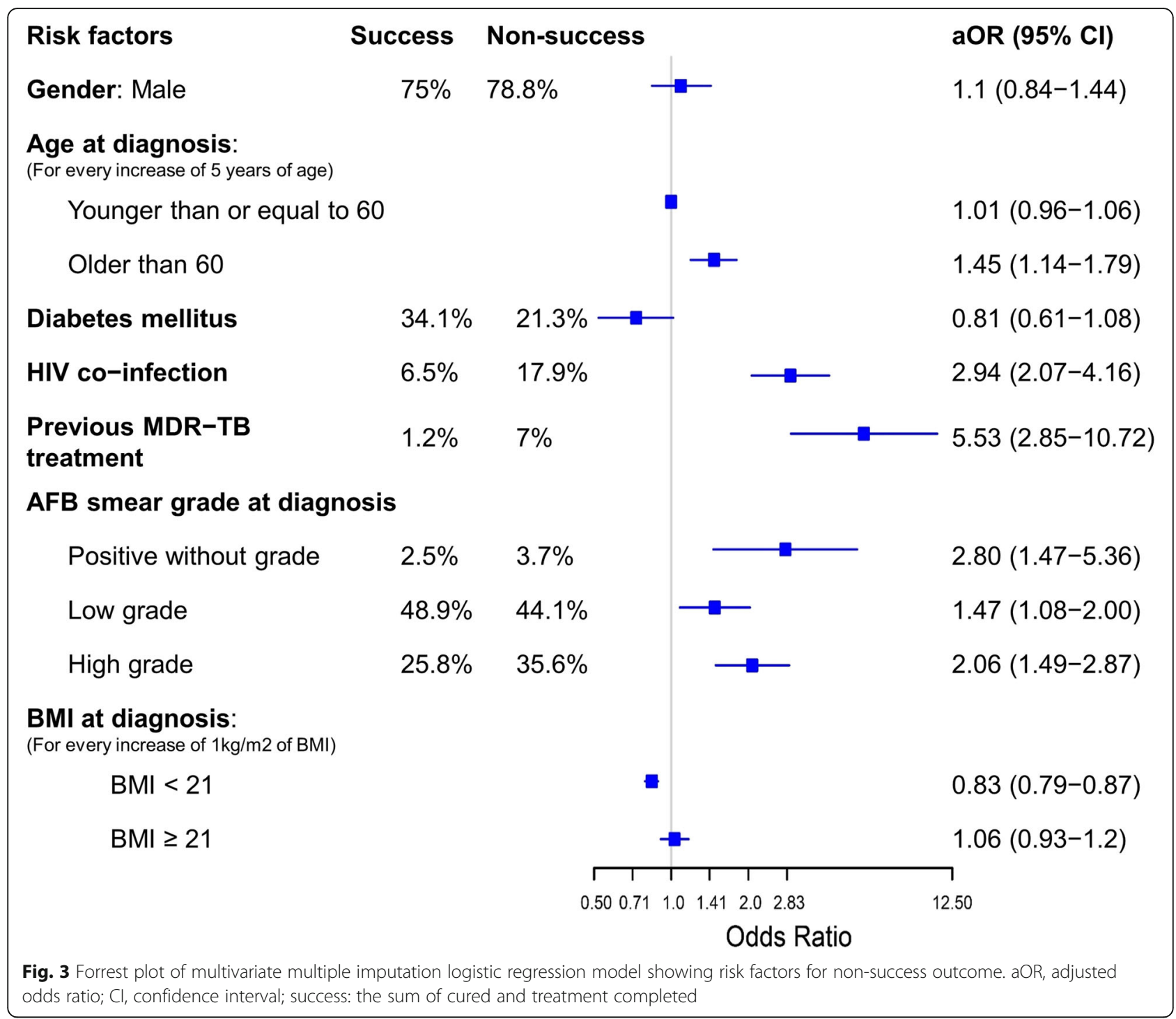

MDR-TB treatment was influenced by the use of metformin, a hypoglycemic agent that might improve TB treatment outcomes [30, 31]. As markers of glycemic control among patients with MDR-TB and DM were unavailable, the effect on treatment outcome could not be assessed. Despite these limitations, DM is a common but neglected comorbidity in MDR-TB patients and should be screened for prior MDR-TB treatment.

This study has several limitations. This is a retrospective study and some records were irretrievable at the study time. Demographic information and records of smear, culture and DST was not completely recorded on the electronic database. The majority of patients (78.4\%) did not have DST results, and we could not include drug resistance information into multivariate logistic regression models. Finally, the information on smoking and alcohol use were not available in our cohort, although they are known risk factors for poor outcome [32] [33]. Therefore, a prospective study is necessary to provide a comprehensive assessment of risk factors for poor treatment outcome in MDR-TB.

\section{Conclusion}

Despite these limitations, the present study emphasizes the increasing trend of MDR-TB in HCMC between 2011 and 2015 and the need for drug resistance screening for all TB patients. Patients with HIV, high smear grade, malnutrition and history of previous MDR-TB treatment are at high risk of poor outcomes. 


\section{Supplementary information}

Supplementary information accompanies this paper at https://doi.org/10. 1186/s12879-020-4887-1

Additional file 1. Details on treatment regimens and outcomes of MDR-TB patients.

Additional file 2. Dataset of 2266 MDR-TB patients.

Additional file 3. Dataset of drug-susceptibility testing results.

\section{Abbreviations}

AFB: Acid-fast bacilli; BMI: Body mass index; DM: Diabetes mellitus; DST: Drug susceptibility testing; DTU: District tuberculosis unit; HCMC: Ho Chi Minh City; IQR: Interquartile range; LPA: Line probe assay; MDR: Multidrug resistant tuberculosis; OR: Odds ratio; PMDT: Programmatic Management of Drugresistant Tuberculosis; PNTH: Pham Ngoc Thach Hospital; RR: Rifampicin resistant; TB: Tuberculosis; XDR: Extensively drug resistance

\section{Acknowledgements}

We would like to thank Le Van Ngo, Tran Minh Khuyen, Pham Thi Thuy Lieu, Nguyen Ngoc Phat, Nguyen Van Hoi, Nguyen Van Thom, Nguyen Van Nghia, Phan Hong Liem, Ly Hong An, Chung Thi Bich Dao, Hoang Van Thang, Nguyen Thi Nguyet Thu, Truong Ngoc Dai, Nguyen Chi Thien, Nguyen Hong Nguyen, Vo Van Tam, Nguyen Trung Hoa, La Duc Du, Nguyen Hoang Tu, Tran Dinh Thu, Tran Quynh Ly, Tran Thi Mai An, Truong Thi Nhu Thuy, Ho Van Nho, Phan Thi Minh Tan, Vo Van Hung and all their staff in 24 DTUs in HCMC; all doctors and nurses of PNTH; Nguyen Lam Vuong for his helpful advice on statistical analysis, and Timothy M. Walker for improving the English of the manuscript.

\section{Authors' contributions}

GT and NTTT conceptualized, designed and revised the manuscript. LHV designed the study, collected, interpreted the data, and draft the manuscript. NHL, TW, NTMT and DTMH participated in the design and data collection. PTP, VTS and NTH coordinated the study and collected the data. DNV and LTHN participated in data analysis and revised manuscript. All authors read and approved the final manuscript.

\section{Funding}

This work was supported by the Wellcome Trust Intermediate Fellowship [206724/Z/17/Z to N.T.T.T] and the Wellcome Trust Major Overseas Program Funding $[106680 / B / 14 / Z$ to G.T]. The funding body has no role in the design of the study, collection, analysis, interpretation of data or in writing the manuscript.

\section{Availability of data and materials}

All data generated or analysed during this study are included in this published article and its supplementary information files in demo.csv and dst.csv.

\section{Ethics approval and consent to participate}

The study was approved by Institutional Review Board (IRB) at PNTH (Reference number: 546/NCKH-PNT) and Oxford Tropical Research Ethics Committee (OxTREC Reference number: 24-17), UK. Individual consent to participate was waived by the IRB because this is a retrospective collection and data were recorded and analyzed anonymously.

\section{Consent for publication}

Not applicable.

\section{Competing interests}

All authors declare no competing interests.

\section{Author details}

${ }^{1}$ Tuberculosis group, Oxford University Clinical Research Unit, 764 Vo Van Kiet street, District 5, Ho Chi Minh City, Vietnam. ${ }^{2}$ Pham Ngoc Thach Hospital, Ho Chi Minh City, Viet Nam. ${ }^{3}$ Centre for Tropical Medicine and Global Health, Nuffield Department of Medicine, University of Oxford, Oxford, UK.
Received: 17 July 2019 Accepted: 13 February 2020

Published online: 22 February 2020

\section{References}

1. Global tuberculosis report 2018 Geneva: World Health Organization; [updated 2018]. Available from: http://www.who.int/tb/publications/global_ report/en/. cited 2019 April

2. Phuong NT, Nhung NV, Hoa NB, Thuy HT, Takarinda KC, Tayler-Smith K, et al Management and treatment outcomes of patients enrolled in MDR-TB treatment in Viet Nam. Public Health Action. 2016;6(1):25-31.

3. Aibana O, Bachmaha M, Krasiuk V, Rybak N, Flanigan TP, Petrenko V, et al. Risk factors for poor multidrug-resistant tuberculosis treatment outcomes in Kyiv oblast, Ukraine. BMC Infect Dis. 2017;17(1):129.

4. Kurbatova EV, Taylor A, Gammino VM, Bayona J, Becerra M, Danilovitz $M$, et al. Predictors of poor outcomes among patients treated for multidrug-resistant tuberculosis at DOTS-plus projects. Tuberculosis. 2012;92(5):397-403.

5. van Altena R, de Vries G, Haar CH, de Lange WC, Magis-Escurra C, van den Hof $\mathrm{S}$, et al. Highly successful treatment outcome of multidrugresistant tuberculosis in the Netherlands, 2000-2009. Int JTuberc Lung Dis. 2015;19(4):406-12.

6. Brust JC, Gandhi NR, Carrara H, Osburn G, Padayatchi N. High treatment failure and default rates for patients with multidrug-resistant tuberculosis in KwaZulu-Natal, South Africa, 2000-2003. Int J Tuberc Lung Dis. 2010;14(4): 413-9.

7. Nhung NV, Hoa NB, Sy DN, Hennig CM, Dean AS. The fourth national antituberculosis drug resistance survey in Viet Nam. Int J Tuberc Lung Dis. 2015; 19(6):670-5.

8. Hoa NB, Khanh PH, Chinh NV, Hennig CM. Prescription patterns and treatment outcomes of MDR-TB patients treated within and outside the National Tuberculosis Programme in Pham Ngoc Thach hospital, Viet Nam. Trop Med Int Health. 2014;19(9):1076-81.

9. Technical manual for drug susceptibility testing of medicines used in the treatment of tuberculosis. Geneva: World Health Organization; 2018 [updated 2018]. Available from: https://www.who.int/tb/publications/2018/ WHO_technical_drug_susceptibility_testing/en/. cited 2019 March

10. Nunn AJ, Rusen ID, Van Deun A, Torrea G, Phillips PP, Chiang CY, et al. Evaluation of a standardized treatment regimen of anti-tuberculosis drugs for patients with multi-drug-resistant tuberculosis (STREAM): study protocol for a randomized controlled trial. Trials. 2014;15:353.

11. Team RC. R: A language and environment for statistical computing. Vienna: R Foundation for Statistical Computing; 2015.

12. Organization $\mathrm{WH}$. Companion handbook to the $\mathrm{WHO}$ guidelines for the programmatic management of drug-resistant tuberculosis 2014. Available from: https://www.who.int/tb/publications/pmdt_companionhandbook/en/. [cited 2019 March]

13. Stef van Buuren KG-O. mice: Multivariate Imputation by Chained Equations in R. Journal of Statistical Software. 2011:2018(August):1-67.

14. Mai TQ, Martinez E, Menon R, Van Anh NT, Hien NT, Marais BJ, et al. Mycobacterium tuberculosis drug resistance and transmission among human immunodeficiency virus-infected patients in Ho Chi Minh City, Vietnam. Am J Trop Med Hyg. 2018;99(6):1397-406.

15. Nhu NT, Lan NT, Phuong NT, Chau N, Farrar J, Caws M. Association of streptomycin resistance mutations with level of drug resistance and Mycobacterium tuberculosis genotypes. Int J Tuberc Lung Dis. 2012;16(4): 527-31

16. Hang NT, Maeda S, Lien LT, Thuong PH, Hung NV, Thuy TB, et al. Primary drug-resistant tuberculosis in Hanoi, Viet Nam: present status and risk factors. PLoS One. 2013:8(8):e71867.

17. Kwak N, Kim HR, Yoo CG, Kim YW, Han SK, Yim JJ. Multidrug-resistant tuberculosis over 20 years at a referral hospital in South Korea: trends and outcomes. Int J Tuberc Lung Dis. 2019;23(2):174-80.

18. Gunther G, van Leth F, Alexandru S, Altet N, Avsar K, Bang D, et al. Clinical Management of Multidrug-Resistant Tuberculosis in 16 European countries. Am J Respir Crit Care Med. 2018;198(3):379-86.

19. World Health Organization treatment guidelines for drug-resistant tuberculosis 2016 update Geneva: World Health Organization; 2016 [cited 2019 March]. Available from: https://apps.who.int/iris/bitstream/handle/1 0665/250125/9789241549639-eng.pdf.

20. Nguyen HB, Nguyen NV, Tran HT, Nquyen HV, Bui QT. Prevalence of resistance to second-line tuberculosis drug among multidrug-resistant 
tuberculosis patients in Viet Nam, 2011. Western Pac Surveill Response J : WPSAR. 2016;7(2):35-40.

21. Vu DH, van Rein N, Cobelens FG, Nguyen TT, Le VH, Brouwers JR. Suspected tuberculosis case detection and referral in private pharmacies in Viet Nam. Int J Tuberc Lung Dis. 2012;16(12):1625-9.

22. Hoa NB, Phuc PD, Hien NT, Hoa VQ, Thuong PH, Anh PT, et al. Prevalence and associated factors of diabetes mellitus among tuberculosis patients in Hanoi, Vietnam. BMC Infect Dis. 2018;18(1):603.

23. Guariguata L, Whiting DR, Hambleton I, Beagley J, Linnenkamp U, Shaw JE. Global estimates of diabetes prevalence for 2013 and projections for 2035. Diabetes Res Clin Pract. 2014;103(2):137-49.

24. Magee MJ, Kempker RR, Kipiani M, Gandhi NR, Darchia L, Tukvadze N, et al. Diabetes mellitus is associated with cavities, smear grade, and multidrugresistant tuberculosis in Georgia. Int J Tuberc Lung Dis. 2015;19(6):685-92.

25. Baker MA, Harries AD, Jeon CY, Hart JE, Kapur A, Lonnroth K, et al. The impact of diabetes on tuberculosis treatment outcomes: a systematic review. BMC Med. 2011;9:81.

26. Salindri AD, Kipiani M, Kempker RR, Gandhi NR, Darchia L, Tukvadze N, et al. Diabetes Reduces the Rate of Sputum Culture Conversion in Patients With Newly Diagnosed Multidrug-Resistant Tuberculosis. Open forum Infecti Dis. 2016;3(3):ofw126.

27. Alarcon V, Alarcon-Arrascue E, Mendoza-Ticona A, Obregon G, Cornejo t, Vargas $D$, et al. Programmatic management of patients with pre-extensively drug-resistant tuberculosis in Peru, 2011-2014. Int J Tuberc Lung Dis. 2018; 22(10):1220-6.

28. Kang YA, Kim SY, Jo KW, Kim HJ, Park SK, Kim TH, et al. Impact of diabetes on treatment outcomes and long-term survival in multidrug-resistant tuberculosis. Respiration; Int Rev Thorac Dis. 2013;86(6):472-8.

29. Johnston JC, Shahidi NC, Sadatsafavi M, Fitzgerald JM. Treatment outcomes of multidrug-resistant tuberculosis: a systematic review and meta-analysis. PLoS One. 2009;4(9):e6914.

30. Singhal A, Jie L, Kumar P, Hong GS, Leow MK, Paleja B, et al. Metformin as adjunct antituberculosis therapy. Sci Transl Med. 2014;6(263):263ra159.

31. Degner NR, Wang JY, Golub JE, Karakousis PC. Metformin use reverses the increased mortality associated with diabetes mellitus during tuberculosis treatment. Clin Infecti Dis. 2018;66(2):198-205.

32. Miller AC, Gelmanova IY, Keshavjee S, Atwood S, Yanova G, Mishustin S, et al. Alcohol use and the management of multidrug-resistant tuberculosis in Tomsk, Russian Federation. Int J Tuberc Lung Dis. 2012;16(7):891-6.

33. Fan YM, Ding SP, Bao ZJ, Wu LM, Zhen LB, Xia Q, et al. Prognostic factors for treatment success in patients with multidrug-resistant tuberculosis in China. Int J Tuberc Lung Dis. 2018;22(3):300-5.

\section{Publisher's Note}

Springer Nature remains neutral with regard to jurisdictional claims in published maps and institutional affiliations.

Ready to submit your research? Choose BMC and benefit from:

- fast, convenient online submission

- thorough peer review by experienced researchers in your field

- rapid publication on acceptance

- support for research data, including large and complex data types

- gold Open Access which fosters wider collaboration and increased citations

- maximum visibility for your research: over $100 \mathrm{M}$ website views per year

At $\mathrm{BMC}$, research is always in progress.

Learn more biomedcentral.com/submissions 